\title{
Attitude (Acceptance) of the COVID-19 Vaccine among Adult Kuwait Oil Company Workers
}

\author{
Zouheir Ibrahim Bitar ${ }^{1}$, Mubarak Juwaied Al-Ajmi' ${ }^{2}$, Ossama Sajeh Maadarani ${ }^{1}$, \\ Yacob Al Shammari ${ }^{3}$, Mahmoud Elzoueiry', Mohammad Al-Muwaizri ${ }^{4}$ \\ ${ }^{1}$ Internal Medicine Department, Ahmadi Hospital, Kuwait Oil Company, Ahmadi, Kuwait \\ ${ }^{2}$ Internal Medicine, Ahmadi Hospital, Ahmadi, Kuwait \\ ${ }^{3}$ Ahmadi Hospital, Kuwait Oil Company, Ahmadi, Kuwait \\ ${ }^{4}$ Nursing Department, Ahmadi Hospital, Kuwait Oil Company, Ahmadi, Kuwait \\ Email: ^zbitar@kockw.com, ossamamaadarani@yahoo.com, respolung@hotmail.com,mmuwaizri@kockw.com
}

How to cite this paper: Bitar, Z.I., Al-Ajmi, M.J., Maadarani, O.S., Al Shammari, Y., Elzoueiry, M. and Al-Muwaizri, M. (2021) Attitude (Acceptance) of the COVID-19 Vaccine among Adult Kuwait Oil Company Workers. Open Journal of Internal Medicine, 11, 210-219.

https://doi.org/10.4236/ojim.2021.114017

Received: April 9, 2021

Accepted: November 27, 2021

Published: November 30, 2021

Copyright $\odot 2021$ by author(s) and Scientific Research Publishing Inc. This work is licensed under the Creative Commons Attribution International License (CC BY 4.0).

http://creativecommons.org/licenses/by/4.0/ (c) (i) Open Access

\begin{abstract}
Objective: To measure the acceptance of a coronavirus disease 2019 (COVID-19) vaccine among the sector of the oil company adult population in Kuwait and assess its determinants. Subjects and Methods: A cross-sectional email study enrolled adults working for Kuwait oil companies ( $n=1689$; aged $\geq 21$ years). Acceptance of a COVID-19 vaccine was considered if participants selected the options and received the vaccine, definitely or probably will accept vaccination against COVID-19 once a vaccine is available." A modified Poisson regression was used to investigate the associations and calculate the adjusted prevalence ratios (aPRs) and their 95\% confidence intervals (CIs). Results: In total, 92.5\% (1563/1689) of the participants were willing to accept COVID-19 vaccines once available. No difference in sex was observed in the willingness to accept a COVID-19 vaccine (92.5 vs. $92 \%$, p 0.5). Responders who believed vaccines in general to have health-related risks were less likely to get vaccinated ( $\mathrm{aPR}=0.39,95 \% \mathrm{CI}: 0.35-0.44)$. In addition, participants who received the influenza vaccine were more willing to have a COVID-19 vaccine (aPR $=1.44,95 \% \mathrm{CI}: 1.31-1.58)$. Acceptance of the vaccine against COVID-19 increased as the self-perceived chances of contracting the infection increased $(p<0.001)$. Conclusion: Overall, $92.5 \%$ of the study participants demonstrated a willingness to be vaccinated against COVID-19. However, we found that public health authorities should address several factors influencing the level of acceptance.
\end{abstract}

\section{Keywords}

COVID-19, Vaccination, Poll 


\section{Introduction}

An outbreak of novel coronavirus in December 2019, known as severe acute respiratory syndrome coronavirus 2 (SARS-CoV-2), occurred in Wuhan city China. As a result, the World Health Organization named the disease coronavirus disease 2019 (COVID-19). The virus caused a cluster of fatal pneumonia cases and rapidly spread worldwide, and subsequently, the WHO declared COVID-19 as a pandemic in March 2020 [1].

Vaccines to prevent SARS-CoV-2 infection are considered the most promising approach for controlling the pandemic and are being vigorously investigated and produced. By the end of 2020, several vaccines had become available for use in different parts of the world [2]. In addition, the World Health Organization maintains an updated list of vaccine candidates under evaluation and that given approval [2]. But misinformation, concerns about safety, loss of trust in vaccine manufacturing, and conspiracy theories surrounding COVID-19 vaccines can be obstacles to vaccine uptake.

Vaccine hesitancy (delay in acceptance or refusal of vaccination despite the availability of vaccination services) is believed to be responsible for decreasing vaccine coverage and an increased risk of vaccine-preventable disease outbreaks and epidemics [3]. However, little is known about the hesitancy and acceptance of the COVID-19 vaccine and the factors that influence its acceptance. Such information is important in planning strategies to decrease hesitancy and increase vaccine acceptability. Therefore, the current survey sought to assess the acceptance of the available COVID-19 vaccine and evaluate factors that can overcome hesitancy and influence its acceptance among the Kuwait Oil Company workers.

\section{Subjects and Methods}

We conducted a cross-sectional study and enrolled adults working in the Kuwait Oil Company (KOC) ( $n=1689$; aged $\geq 21$ years) using an email survey distributed to $3000 \mathrm{KOC}$ staff using the official KOC email platforms. The enrollment of subjects started on June 1st, 2021, for four weeks. We used the voluntary response sample to recruit participants, a nonprobability sampling method which yields a convenience sample. The email survey was distributed three times/week to KOC staff through the official institute email system. The study was approved by the KOC administration and Ahmadi Hospital management. Completion of the questionnaire was optional, and those who provided consent replied to the email.

\section{Study Questionnaire and Variable Definitions}

The study questionnaire was designed to be completed by the KOC employee and submitted through the same email, with information on sociodemographic data and lifestyle factors gathered (Table 1).

The responders reported a personal history of COVID-19 diagnosis. Subsequently, participants were asked about whether they had received the vaccine, 
Table 1. Study questionnaire and variable definitions.

Kuwait Oil Company view of vaccine COVID-19

\section{Uptake}

When [the coronavirus vaccine] becomes available to you, will you ...?

acceptance

Get vaccine (Offered and vaccinated)

Definitely get it

Probably get it

refusal

Not get vaccine

Probably not get it

Definitely not get it

\section{Efficacy}

- Among those who said they plan to get a vaccine against the coronavirus when it becomes available: Which of the following are reasons you would get a coronavirus vaccine?

I want to protect my family

I want to protect myself

It would be the best way to avoid getting seriously ill from the coronavirus

I want to protect my community

It would allow me to feel safe around other people

Life won't go back to normal until most people are vaccinated

I have confidence in the vaccine development and approval process

It would allow me to go back to normal activities like work or school

My doctor recommends vaccines

I have a chronic health condition, such as asthma or diabetes, so it is important that I receive a coronavirus vaccine

\section{Safety}

- Among those who said they do not plan to get a vaccine against the coronavirus when it becomes available: Which of the following are reasons you would not get a coronavirus vaccine?

I would be concerned about side effects from the vaccine

I'm concerned about the development and approval process

I would be concerned about getting infected with the coronavirus from the vaccine

I'm not concerned about getting seriously ill from the coronavirus

The coronavirus outbreak is not as serious as some people say it is

I don't think vaccines work very well

I don't like needles

I am allergic to vaccines

I won't have time to get vaccinated

\section{Trust in Sources of Vaccine Information}

How much do you trust each of the following sources for information about coronavirus vaccines? 


\section{Continued}

Health professionals, including doctors, nurses, and pharmacists

Ministry of health

CDC

FDA

HHS

WHO

Pharmaceutical companies

News media

HHS Department of Health and Human Services; CDC Centers for Disease Control and Prevention; FDA Food and Drug Administration; WHO World Health Organization.

their willingness to receive a potential COVID-19 vaccine once available in general ("definitely yes", "probably yes", "get the vaccine", "not get the vaccine", "probably no", and "definitely no").

The general acceptance of a COVID-19 vaccine was our primary outcome variable, which was dichotomized as acceptance (received the vaccine, "definitely and probably yes",) and refusal ("definitely and probably no, will not get vaccinated").

Subjects who selected the option to get a vaccine against the coronavirus when it becomes available can choose more than one option: Which of the following reasons would you get a coronavirus vaccine? I want to protect my family. I want to protect myself. It would be a way to avoid getting seriously ill from the coronavirus. I want to protect my community. It would allow me to feel safe around other people. Life will not go back to normal until most people are vaccinated. I have trust in the vaccine development and approval process. It would allow me to go back to regular activities, like work or sports. My doctor recommends vaccines. I have a chronic health condition, such as chronic renal failure or diabetes, so I must receive a coronavirus vaccine.

For the subjects who were selected, they will not get the vaccine against the coronavirus when it becomes available, which of the following are their reasons for not getting a coronavirus vaccine. I would be concerned about the side effects from the vaccine. I am concerned about the development and approval process. I am concerned about getting infected with the coronavirus from the vaccine. I am not concerned about getting seriously ill from the coronavirus. The coronavirus outbreak is not as serious as some people say it is. I do not think vaccines work very well. I do not like needles. I am allergic to vaccines. I will not have time to get vaccinated.

Among those who said they definitely or would probably not get a coronavirus vaccine that was determined to be safe by scientists and was available for free to everyone who wanted it, they were asked the following: Please tell me if each of the following is a major reason, a minor reason, or not a reason why you 
would [probably] NOT get a COVID-19 vaccine: Worried about possible side effects; do not trust the government to make sure the vaccine is safe and protective; the vaccine is too new and wants to wait and see how it affects life of other people; politics has played too much of a role in the vaccine development process; the risks of COVID-19 are being exaggerated; do not trust vaccines in general; do not trust the health authorities; worried that they may get COVID-19 from the vaccine; do not think they are at risk of getting sick from COVID-19.

Among those who said they probably would not or definitely would not get the COVID-19 Vaccine: How much did the factor of the following reasons into your decision: Concern about side effects, want to know more about how well it works; do not think I need it; it would cost too much.

\section{Results}

\subsection{Distribution of Demographic Characteristics}

In total, 1689 adults (442 females) completed the study questionnaire, among whom 66 (3.9\%) reported having suffered from COVID-19. Most participants were aged 36 (median from 21 and 67) (25.6\%) years, with 697 of Kuwaiti nationality (Table 2). Health care workers in Kuwait Oil Company accounted for 246 of the total participants, and $8.7 \%$ of the participants were physicians.

\subsection{The Demographic Characteristics}

There was no difference in sex in terms of accepting vaccination against COVID-19 (92.5\% vs. 92\%, p 0.5; Figure 1 ). Noncitizens were more likely to accept a COVID-19 vaccine than national citizens (96\% vs. $87 \%, p<0.001$ ). Physicians were the most likely group to accept vaccination against COVID-19 (75.6\%).

\subsection{Factors Associated with the Acceptance of a COVID-19 Vaccine}

The factors that most strongly correlated with the acceptance of the vaccine were as follows (Table 3): "It would allow me to feel safe around other people (13\%)", "I want to protect my community (17\%)", "I want to protect my community (17\%)", "I want to avoid getting seriously ill from the coronavirus (16\%)", "I want to protect myself (17\%)", and "I want to protect my family (18\%)".

Participants who assumed that they were highly likely to contract the infection

Table 2. Demographic.

\begin{tabular}{cc}
\hline variable & N (\%) \\
\hline Female & $442(26)$ \\
Citizen & $697(41)$ \\
Age (median) & $36(21-67)$ \\
Healthcare worker & $246(15)$ \\
\hline
\end{tabular}


Table 3. Kuwait oil company view of vaccine COVID-19.

1) Uptake: When (the coronavirus vaccine) becomes available to you, will you ...? N (\%)

Definitely not get it

$38(2)$

Probably not get it

Not get vaccine

Probably get it

$96(6)$

Definitely get it

Get vaccine $747(44)$

2) Efficacy: Among those who said they plan to get a vaccine against the coronavirus when it becomes available: Which of the following are reasons you would get a coronavirus vaccine? $\mathrm{N}(\%)$

I have confidence in the vaccine development and approval process $66(1)$

I have a chronic health condition, such as asthma or diabetes, so it is important that I receive a coronavirus vaccine

My doctor recommends vaccines

Life won't go back to normal until most people are vaccinated

It would allow me to go back to normal activities like work or school

It would allow me to feel safe around other people

I want to protect my community

$1072(17)$

3) Safety: Among those who said they do not plan to get a vaccine against the coronavirus when it becomes available: Which of the following are reasons you would not get a coronavirus vaccine? Percentage. N (\%)

I won't have time to get vaccinated

I am allergic to vaccines

I don't like needles

The coronavirus outbreak is not as serious as some people say it is

I'm not concerned about getting seriously ill from the coronavirus

I would be concerned about getting infected with the coronavirus from the vaccine

I don't think vaccines work very well

I'm concerned about the development and approval process

410 (18)

I would be concerned about side effects from the vaccine

$1056(47)$

I won't have time to get vaccinated

$32(1)$

I am allergic to vaccines

I don't like needles

The coronavirus outbreak is not as serious as some people say it is

I'm not concerned about getting seriously ill from the coronavirus

$120(5)$

Q4 Trust in Sources of Vaccine Information N (\%)

Health professionals, including doctors, nurses, and pharmacists

1155 (68) 


\section{Continued}

\begin{tabular}{ll}
\hline Ministry of health & $1185(70)$ \\
CDC & $938(56)$ \\
FDA & $907(54)$ \\
HHS & $764(45)$ \\
WHO & $948(56)$ \\
Pharmaceutical companies & $395(23)$ \\
News media & $289(17)$ \\
\hline
\end{tabular}

HHS Department of Health and Human Services; CDC Centers for Disease Control and Prevention; FDA Food and Drug Administration; WHO World Health Organization.

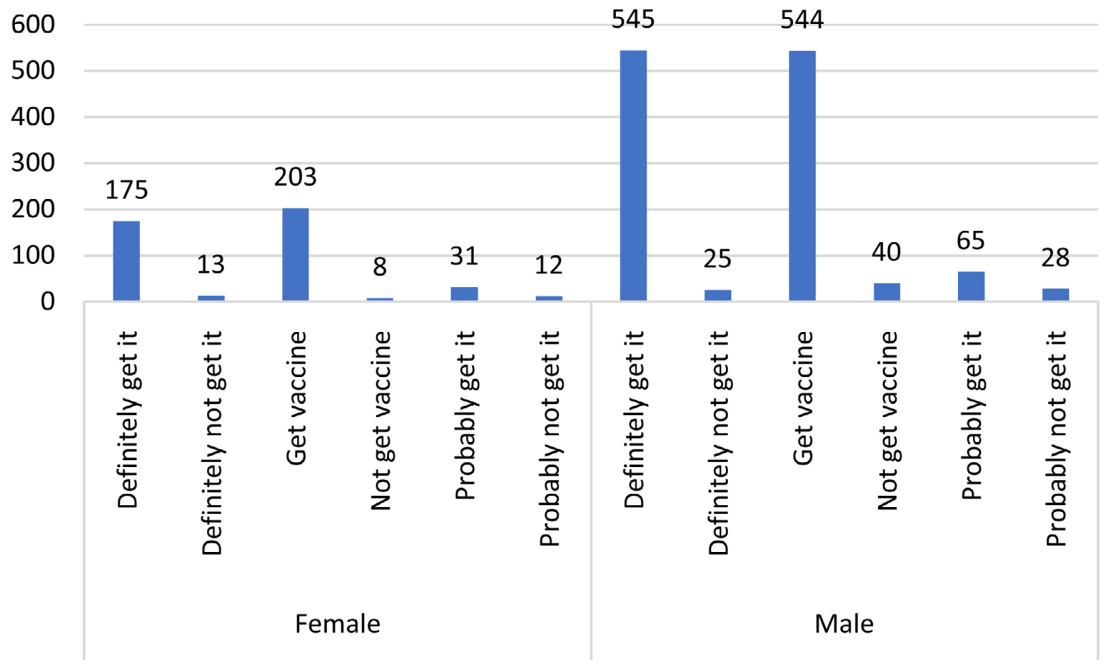

Figure 1. Acceptance of a COVID-19 vaccine according to gender.

were more accepting of a vaccine than those who thought they were unlikely to contract the infection $(88 \%$ vs. $35.4 \%, p$ trend $<0.001)$. According to the belief of severity of the disease, participants who believe that their COVID-19 disease would be mild were less agreeing on the vaccine than those who anticipated that their disease complication would be severe $(50.2 \%$ vs. $77.9 \%$, $p$ trend $<0.001)$.

Moreover, subjects who agreed with "It would be the best way to avoid getting seriously ill from the coronavirus" were more willing to accept a COVID-19 vaccine than those who agreed with the development of natural immunity following the disease $(69.1 \%$ vs. $34.2 \%, p$ trend $<0.001)$.

Moreover, participants who indicated that vaccines protect against infectious diseases were more likely to accept a potential COVID-19 vaccine (aPR: 5.67; 95\% CI: 4.14 - 7.77). Conversely, participants who viewed vaccines to have health-related risks were less willing to accept a potential COVID-19 vaccine (aPR: 0.39; 95\% CI: 0.35 - 0.44).

\subsection{Concerns Related to a COVID-19 Vaccine}

In the total study sample, the majority of the participants were most concerned 
about possible side effects (47\%), concerned about the development and approval process (18\%), and doubtful of its efficacy (10\%) (Table 3). In general, responders who were willing to accept a COVID-19 vaccine demonstrated less concern than subjects who refused vaccination against COVID-19.

Trust in sources of vaccine information is important in accepting the idea of vaccination. Health professionals (68\%) and the Ministry of Health Media (70\%) were the most trusted sources; at the bottom of the list, social media accounted for $17 \%$, and pharmaceutical companies accounted for $23 \%$.

\section{Discussion}

The potential of vaccines to stop the transmission of COVID-19 pneumonia and reach community immunity depends not only on the efficacy of the vaccine but also on the public's willingness to be vaccinated. Maintaining interest in vaccination among the public is a long-term challenge. In this poll, we found that $44 \%$ received the vaccine, and $43 \%$ will get the vaccine once available. Such a level of acceptability is considerably high compared to earlier studies during the COVID-19 pandemic [4].

The percentage of acceptance of a COVID-19 vaccine among our study participants (87\%) was lower than that among Malaysian residents (94.3\%) [5] and adults in the United States ( 70\%) [6]. The acceptance of vaccines varied with time; in Kuwait, it was 43\% in August 2020 [7], and in our study, it was $92.5 \%$ in June. For those willing to get the vaccine for a reason, 35\% responded that they wanted to protect themselves, family, and their community. In addition, 9\% responded that the vaccine allowed them to return to normal life (work, school, restaurants, and traveling), given laws in Kuwait where nonvaccinated individuals are not allowed to travel or attend common places such as malls and restaurants.

Although the adoption of the COVID-19 vaccine in Kuwait indicates that assumed effectiveness is an important motivator, the poll findings warn that public perceptions of effectiveness may differ from investigator views. The public may expect that an effective COVID-19 vaccine means immediate changes in their daily experience and not only strong protection against the virus. Indeed, when asked about the reasons they would take the vaccine, many responders stated it would let them "to go back to normal activities like work or school" (9\%) or "feel safe around other people" (13\%) and protect family (17\%). Such believes may conflict with expert warnings that get a vaccine, regardless of how effective it may be in preventing infection, does not guarantee a return to normal.

Concern about the possible side effects, the safety of the vaccine, the effectiveness of vaccination against COVID-19, and the development and approval process are the main factors related to refusing a potential COVID-19 vaccine. Such factors have been reported in prior studies [8] [9]. In addition, we demonstrated that individuals who refused vaccination were more likely to express such hesitancy concerns than study participants who reported a willingness to receive 
vaccination against COVID-19 once a vaccine was available. It is a point that should be stressed and explained well when giving information to the public to increase the uptake of a potential COVID-19 vaccine.

Although our study sample was average, it represented only the employees who work in Kuwait oil companies and not people who reside in all areas in Kuwait. Nevertheless, the generalizability of our results is hindered by the questionable representativeness of our study sample because good percentages of people who work cannot access their electronic mail. Furthermore, there were new rules given by the government that pushed people to seek vaccination. The rules were given in preparation to return to normal life, e.g., traveling, entering malls and restaurants, which pushed hesitant people to seek vaccination but were not effective reasons during this study.

\section{Conclusion}

In conclusion, overall, $92.5 \%$ of the study participants demonstrated a willingness to get vaccinated against COVID-19. Our findings are important in state health planning and should help guide public health efforts in escalating the acceptance of COVID-19 vaccination in the population of Kuwait. However, in transparent communication, public health authorities must address the misinformation and conspiracy views surrounding the vaccination against COVID-19 disease. Explaining to the public the safety, effectiveness, and expected complications of different COVID-19 vaccination will contribute to increasing trust in future COVID-19 vaccination programs.

\section{Acknowledgements}

The authors are grateful for the suggestions and help from the manager of medical group KOC Dr. Emad AlAwad and chief clinical officer Dr. Soud AlAjmi that have substantially contributed to the final shape of the paper.

\section{Conflicts of Interest}

The author declares no conflicts of interest regarding the publication of this paper.

\section{References}

[1] World Health Organization (2020) Coronavirus Disease 2019 (Covid-19): Situation Report, 51. World Health Organization, Geneva. https://apps.who.int/iris/handle/10665/331475

[2] World Health Organization (2020) Draft Landscape of COVID-19 Candidate Vaccines. https://www.who.int/publications/m/item/draft-landscape-of-covid-19-candidate-v accines

[3] MacDonald, N.E. and SAGE Working Group on Vaccine Hesitancy (2015) Vaccine Hesitancy: Definition, Scope and Determinants. Vaccine, 33, 4161-4164. https://doi.org/10.1016/j.vaccine.2015.04.036 
[4] Biasio, L.R., Bonaccorsi, G., Lorini, C. and Pecorelli, S. (2021) Assessing COVID-19 Vaccine Literacy: A Preliminary Online Survey. Human Vaccines \& Immunotherapeutics, 17, 1304-1312. https://doi.org/10.1080/21645515.2020.1829315

[5] Wong, L.P., Alias, H., Wong, P.F., Lee, H.Y. and AbuBakar, S. (2020) The Use of the Health Belief Model to Assess Predictors of Intent to Receive the Covid-19 Vaccine and Willingness to Pay. Human Vaccines \& Immunotherapeutics, 16, 2204-2214. https://doi.org/10.1080/21645515.2020.1790279

[6] Malik, A.A., McFadden, S.M., Elharake, J. and Omer, S.B. (2020) Determinants of Covid-19 Vaccine Acceptance in the US. EClinicalMedicine, 26, 100495. https://doi.org/10.1016/j.eclinm.2020.100495

[7] Alqudeimat, Y., Alenezi, D., AlHajri, B., Alfouzan, H., Almokhaizeem, Z., Altamimi, S., Almansouri, W., Alzalzalah, S. and Ziyab, A.H. (2021) Acceptance of a COVID-19 Vaccine and Its Related Determinants among the General Adult Population in Kuwait. Medical Principles and Practice, 30, 262-271. https://doi.org/10.1159/000514636

[8] Reiter, P.L., Pennell, M.L. and Katz, M.L. (2020) Acceptability of a Covid-19 Vaccine among Adults in the United States: How Many People Would Get Vaccinated? Vaccine, 38, 6500-6507. https://doi.org/10.1016/j.vaccine.2020.08.043

[9] Neumann-Bohme, S., Varghese, N.E., Sabat, I., Barros, P.P., Brouwer, W., van Exel, J., et al. (2020) Once We Have It, Will We Use It? A European Survey on Willingness to Be Vaccinated against Covid-19. The European Journal of Health Economics, 21, 977-982. https://doi.org/10.1007/s10198-020-01208-6 